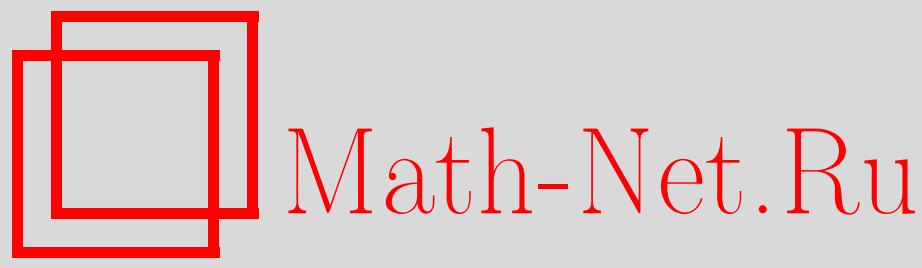

В. А. Авдеев, Локальная сжимаемость процесса изменения рейтинга игрока в модели Эло с одним соперником, Дискрет. матем., 2015, том 27, выпуск 1, 3-21

DOI: https://doi.org/10.4213/dm1311

Использование Общероссийского математического портала Math-Net.Ru подразумевает, что вы прочитали и согласны с пользовательским соглашением http: //www . mathnet.ru/rus/agreement

Параметры загрузки:

IP : 34.229 .108 .108

26 апреля 2023 г., 14:13:07

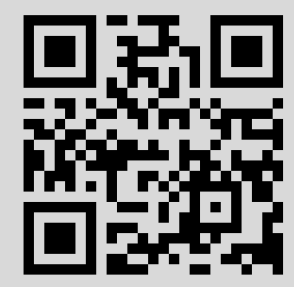




\title{
Локальная сжимаемость процесса изменения рейтинга игрока в модели Эло с одним соперником
}

() 2015 г. В. А. Авдеев*

\begin{abstract}
Рассматривается процесс изменения рейтинга игрока в бесконечной серии игр с одним и тем же соперником в системе Эло вычисления рейтингов. Доказывается существование у этого процесса стационарного распределения и находится верхняя оценка скорости сходимости к нему. В предыдущей статье автора существование предельного распределения было доказано при более жестких предположениях о параметрах способа вычисления рейтинга.
\end{abstract}

Ключевые слова: марковские цепи, итерационные функциональные системы, предельные распределения, локальная сжимаемость, модели рейтинговых систем.

\section{1. Введение}

В данной работе обобщаются теоремы из [1]. Напомним основные понятия, введенные в [1], и полученные в ней результаты.

Как известно, в настоящее время различные рейтинговые системы широко применяются в спорте и онлайн-играх для квалификации игроков на турниры, подбора соперников близкого уровня и в других задачах. Одним из наиболее известных способов вычисления рейтингов игроков в турнирах является система, предложенная в 1960 году Арпадом Эло. Мы рассматриваем ее в упрощенной ситуации, когда рейтинги вычисляются по результатам игр двух игроков друг с другом.

В модели Эло изменение рейтинга игрока за одну партию описывается формулой

$$
R_{\text {new }}=R_{\text {old }}+k\left(W-W_{E}\right),
$$

где $k$ - константа, определяющая динамику изменения рейтинга, $W-$ реально набранное игроком число очков (0 или 1 , то есть дополнительно предполагаем, что в играх не бывает ничьих) и $W_{E}$ - ожидаемое число набранных очков, которое равняется оценке вероятности победить соперника.

Обозначая игроков буквами $A$ и $B$, будем добавлять соответствующий верхний индекс к переменным, относящимся к одному из них.

Пусть в каждой партии сила одного игрока $p^{A}$ имеет нормальное распределение $\mathcal{N}\left(s^{A}, \sigma^{2}\right)$, сила другого, соответственно, $p^{B} \sim \mathcal{N}\left(s^{B}, \sigma^{2}\right)$, и силы игроков в разных партиях независимы.

*Место работы: МГУ им. М.В.Ломоносова, e-mail: avdeev.vadim@gmail.com 
Игрок $A$ будет выигрывать в партии, если случайная величина $p_{A}-p_{B}>0$. Так как $p_{A}-p_{B} \sim \mathcal{N}\left(s_{A}-s_{B}, 2 \sigma^{2}\right)$, то вероятность его победы составит $\mathbf{P}\left\{p_{A}-p_{B}>0\right\}=\Phi\left(\frac{s_{A}-s_{B}}{\sqrt{2} \sigma}\right)$, где $\Phi(x)-$ функция стандартного нормального распределения. Следовательно, в качестве оценки вероятности победы игрока $A$ можно использовать величину $\Phi\left(\frac{R_{\mathrm{old}}^{A}-R_{\mathrm{old}}^{B}}{\sqrt{2} \sigma}\right)$.

Предположим, что игроки $A$ и $B$ в некоторый момент времени имеют рейтинги $R_{0}^{A}$ и $R_{0}^{B}$, после чего начинают бесконечную серию игр только друг с другом. Так как сумма рейтингов игроков после каждой партии остается неизменной, то рейтинг игрока $A$ изменяется по закону

$$
R_{\text {new }}^{A}=R_{\text {old }}^{A}+k W^{A}-k \Phi\left(\frac{2 R_{\text {old }}^{A}-\left(R_{0}^{A}+R_{0}^{B}\right)}{\sqrt{2} \sigma}\right),
$$

где случайная величина $W^{A}=\mathbf{1}_{p^{A}>p^{B}}$ имеет распределение Бернулли $\mathcal{B}(1, q)$ с вероятностью успеха, равной $q=\Phi\left(\frac{s^{A}-s^{B}}{\sqrt{2} \sigma}\right)$.

Обозначая $\frac{2}{\sqrt{2} \sigma}$ через $\alpha$ и $-\frac{R_{0}^{A}+R_{0}^{B}}{\sqrt{2} \sigma}$ через $\beta$, получаем следующую общую модель изменения рейтинга игрока $A$ по результату $n$-й партии:

$$
R_{n}=R_{n-1}-k \Phi\left(\alpha R_{n-1}+\beta\right)+k W_{n},
$$

где все $W_{n}$ независимы и одинаково распределены, $W_{n} \sim \mathcal{B}(1, q), q, k, \alpha, \beta, R_{0}=R_{0}^{A}$ - константы, причем $0<q<1$ и $k, \alpha>0$. При этом в статье [1] дополнительно предполагалось, что $k \leqslant \sqrt{\pi} \sigma=\frac{\sqrt{2 \pi}}{\alpha}$.

Пусть теперь $f(x), x \in \mathbb{R},-$ случайная функция, принимающая значения

$$
\begin{aligned}
& f_{0}(x)=x-k \Phi(\alpha x+\beta), \\
& f_{1}(x)=x-k \Phi(\alpha x+\beta)+k
\end{aligned}
$$

с вероятностями $\{1-q, q\}$ соответственно. Тогда процесс $\left\{R_{n}(x)\right\}$ можно представить следующим образом:

$$
R_{n}(x)=f_{W_{n}}\left(R_{n-1}(x)\right)=\left(f_{W_{n}} \circ \ldots \circ f_{W_{1}}\right)(x),
$$

где все $f_{W_{n}}$ независимы и распределены как $f$. Обозначим данную итерационную функциональную систему через $\mathcal{R}$, а функции $f_{0}(x)$ и $f_{1}(x)$ через $f_{i}(x)$ там, где это не имеет значения.

Кроме того, рассмотрим вспомогательный процесс

$$
\widetilde{R}_{n}(x)=\left(f_{W_{1}} \circ \ldots \circ f_{W_{n}}\right)(x), \quad \widetilde{R}_{0}(x)=x .
$$

В статье [1] на основе результатов [4] и непрерывности функций $f_{0}$ и $f_{1}$ была доказана следующая теорема.

Теорема 1. Пусть предел $\widetilde{R}_{\infty}(x)=\lim _{n \rightarrow+\infty} \widetilde{R}_{n}(x)$ существует почти наверное, конечен и не зависит от $x$. Тогда распределение случайной величины $\widetilde{R}_{\infty}$ является единственным стационарным распределением процесса $\left\{R_{n}(x)\right\}$.

В случае $\alpha k \leqslant \sqrt{2 \pi}$, как легко проверить, обе функции $f_{0}(x)$ и $f_{1}(x)$ строго возрастают, поэтому у них существуют обратные функции, и можно рассмотреть марковский процесс

$$
\widetilde{R}_{n}^{-1}(x)=\left(f_{W_{n}}^{-1} \circ \ldots \circ f_{W_{1}}^{-1}\right)(x) .
$$


Доказав, что для любого $x$ предел $\widetilde{R}_{n}^{-1}(x)$ при $n \rightarrow+\infty$ равняется $\pm \infty$ почти наверное, можно показать, что тогда выполняются условия теоремы 1 , откуда и будет следовать существование единственного стационарного распределения процесса $\left\{R_{n}(x)\right\}$.

В общем случае $\alpha, k>0$, как видно по рисунку 1 , функции $f_{0}(x)$ и $f_{1}(x)$ уже не являются монотонными, но доказать выполнение условий теоремы 1 все равно возможно.
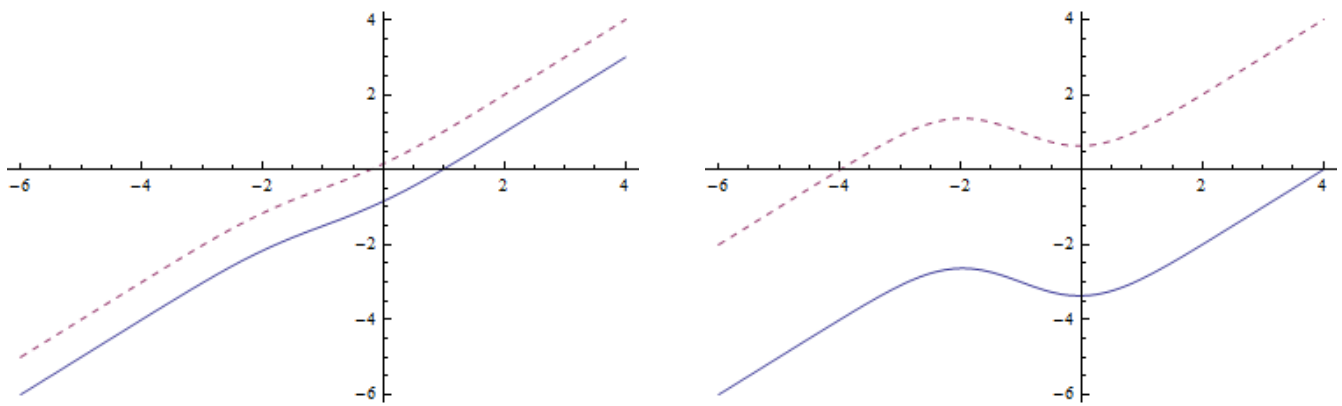

Рис. 1. Функции $f_{0}(x)$ (сплошной линией) и $f_{1}(x)$ (пунктиром) в двух случаях: при $\alpha=1, \beta=1, k=1$ и при $\alpha=1, \beta=1, k=4$.

Для этого введем следующее понятие:

Определение 1. Локальной константой Липшица функции $h$ в точке $x$ называется

$$
L_{x}(h)=\limsup _{y \rightarrow x} \frac{|h(y)-h(x)|}{|y-x|} .
$$

Утверждение 1. Для непрерывно дифференцируемъх функиий $h(x), h_{1}(x)$ u $h_{2}(x)$ верны следующие равенства:

$$
\begin{gathered}
L_{x}(h)=\left|h^{\prime}(x)\right|, \\
L_{x}\left(h_{1} \circ h_{2}\right)=L_{h_{2}(x)}\left(h_{1}\right) L_{x}\left(h_{2}\right) .
\end{gathered}
$$

В частности,

$$
L_{x}(f)=L_{x}\left(f_{i}\right)=\left|1-\frac{\alpha k}{\sqrt{2 \pi}} e^{-\frac{1}{2}(\alpha x+\beta)^{2}}\right| .
$$

Как можно заметить, функция $L_{x}(f)$ может принимать сколь угодно большие значения в окрестности точки $-\frac{\beta}{\alpha}$ при достаточно больших значениях произведения $\alpha k$, поэтому итерационная функциональная система $\mathcal{R}$ не является ни сильно, ни в среднем сжимающей, и к ней не применимы соответствующие стандартные теоремы.

Тем не менее, используя понятие локальной сжимаемости, введенное в работе [5], утверждение о существовании единственного стационарного распределения процесса $\left\{R_{n}(x)\right\}$ можно доказать при общем условии $\alpha, k>0$, в отличие от случая $\alpha k \leqslant \sqrt{2 \pi}$, рассмотренного в статье [1].

Определение 2. Итерационная функциональная система $\mathcal{R}$ называется локально сжимающей, если существует такая функция нормировки $\psi(x): \mathbb{R} \rightarrow[1,+\infty)$, что

$$
\mathbf{E}\left\{L_{x}\left(\widetilde{R}_{n}\right)\right\} \leqslant \psi(x) r^{n}
$$

для некоторого числа $r \in(0,1)$ и всех $x \in \mathbb{R}, n \in \mathbb{N}_{0}$. 
Так как нахождение $L_{x}\left(\widetilde{R}_{n}\right)$ в явном виде затруднительно, в [5] доказано, что для локальной сжимаемости с непрерывной функцией нормировки $\psi(x): \mathbb{R} \rightarrow[1,+\infty)$ достаточно выполнения условия

$$
\sup _{x} \mathbf{E}\left\{\frac{\psi(f(x))}{\psi(x)} L_{x}(f)\right\} \leqslant r<1 .
$$

Доказательство этого утверждения мы проведем позднее, сначала установив выполнение условия (1) для определенным образом выбранной функции $\psi(x)$.

Отметим, что, как утверждается в статье [2], для выбора функции нормировки нет общих методов, более того, в некоторых случаях приходится использовать разные функции нормировки для одних и тех же функций $f_{0}, f_{1}$ при изменении распределения вероятностей $\{1-q, q\}$.

Следствием сложности выбора функции нормировки является невозможность распространить доказательство условия (1) на функции $f(x)$ существенно более общего вида.

Сформулируем основную теорему данной статьи.

Теорема 2. Процесс $\left\{R_{n}(x)\right\}$ имеет единственное стационарное распределение npu $\alpha, k>0$.

Для ее доказательства нам потребуется следующая теорема, справедливость которой мы установим в разделе 3 .

Теорема 3. Для итерационной системы $\mathcal{R}$ условие (1) выполнено с функиией нормировки $\psi(x)=\exp \{c \lambda(x)\}$, где

$$
\lambda(x)=|| x+\frac{\beta}{\alpha}\left|-\frac{k}{2}\right|, \quad c=\frac{\alpha^{2} k}{3\left(1-\frac{\alpha k}{3 \sqrt{2 \pi}} e^{-\frac{9}{8} \alpha^{2} k^{2}}\right)},
$$

и с числом

$$
r=\max \left\{1-\frac{\alpha k}{3 \sqrt{2 \pi}} e^{-\frac{9}{8} \alpha^{2} k^{2}},(1-q) e^{-\frac{1}{3} \alpha^{2} k^{2}}+q\right\}
$$

\section{2. Вспомогательные леммы}

Докажем несколько вспомогательных лемм и утверждений.

Введем следующее обозначение:

$$
\begin{aligned}
\Psi_{q}(x)=\mathbf{E}\left\{\frac{\psi(f(x))}{\psi(x)} L_{x}(f)\right\}= & \\
=(1-q) \frac{\psi\left(f_{0}(x)\right)}{\psi(x)} L_{x}\left(f_{0}\right) & +q \frac{\psi\left(f_{1}(x)\right)}{\psi(x)} L_{x}\left(f_{1}\right)= \\
& =\frac{L_{x}(f)}{\psi(x)}\left((1-q) \psi\left(f_{0}(x)\right)+q \psi\left(f_{1}(x)\right)\right) .
\end{aligned}
$$

Лемма 1. $\Psi_{q}(x)=\Psi_{1-q}\left(-\frac{2 \beta}{\alpha}-x\right)$.

Доказательство. Функция $\lambda(x)$ симметрична относительно $-\frac{\beta}{\alpha}$ :

$$
\lambda\left(-\frac{2 \beta}{\alpha}-x\right)=||-\frac{2 \beta}{\alpha}-x+\frac{\beta}{\alpha}\left|-\frac{k}{2}\right|=|| x+\frac{\beta}{\alpha}\left|-\frac{k}{2}\right|=\lambda(x),
$$


поэтому $\psi\left(-\frac{2 \beta}{\alpha}-x\right)=\psi(x)$.

Функция $L_{x}(f)$ также симметрична относительно этой точки:

$$
L_{-\frac{2 \beta}{\alpha}-x}(f)=\left|1-\frac{\alpha k}{\sqrt{2 \pi}} e^{-\frac{1}{2}(-2 \beta-\alpha x+\beta)^{2}}\right|=\left|1-\frac{\alpha k}{\sqrt{2 \pi}} e^{-\frac{1}{2}(\alpha x+\beta)^{2}}\right|=L_{x}(f) .
$$

Так как $\Phi(-x)=1-\Phi(x)$, то

$$
\begin{aligned}
\left|f_{0}\left(-\frac{2 \beta}{\alpha}-x\right)+\frac{\beta}{\alpha}\right|=\left|-\frac{2 \beta}{\alpha}-x-k \Phi(-2 \beta-\alpha x+\beta)+\frac{\beta}{\alpha}\right|= \\
\quad=\left|x+k \Phi(-\alpha x-\beta)+\frac{\beta}{\alpha}\right|=\left|x-k \Phi(\alpha x+\beta)+k+\frac{\beta}{\alpha}\right|=\left|f_{1}(x)+\frac{\beta}{\alpha}\right|
\end{aligned}
$$

и аналогично $\left|f_{1}\left(-\frac{2 \beta}{\alpha}-x\right)+\frac{\beta}{\alpha}\right|=\left|f_{0}(x)+\frac{\beta}{\alpha}\right|$, поэтому при $i=0,1$

$$
\lambda\left(f_{i}\left(-\frac{2 \beta}{\alpha}-x\right)\right)=\lambda\left(f_{1-i}(x)\right)
$$

и, следовательно, $\psi\left(f_{i}\left(-\frac{2 \beta}{\alpha}-x\right)\right)=\psi\left(f_{1-i}(x)\right)$.

Таким образом, окончательно имеем:

$$
\Psi_{1-q}\left(-\frac{2 \beta}{\alpha}-x\right)=\frac{L_{x}(f)}{\psi(x)}\left(q \psi\left(f_{1}(x)\right)+(1-q) \psi\left(f_{0}(x)\right)=\Psi_{q}(x),\right.
$$

что и требовалось.

Из леммы 1 следует, что для доказательства оценки (1) в нашем случае достаточно рассмотреть только $x \in\left[-\frac{\beta}{\alpha},+\infty\right)$.

Лемма 2. При всех положительных $\alpha$ u $k$ определим числа $x_{3} \quad$ u $x_{4}$ как максимальные корни уравнений $f_{0}\left(x_{3}\right)=-\frac{\beta}{\alpha} u f_{0}\left(x_{4}\right)=-\frac{\beta}{\alpha}+\frac{k}{2}$ соответственно. Тогда

$$
\begin{gathered}
-\frac{\beta}{\alpha}+\frac{k}{2}<x_{3}<-\frac{\beta}{\alpha}+k<x_{4}<-\frac{\beta}{\alpha}+\frac{3 k}{2} \\
f_{0}\left(-\frac{\beta}{\alpha}\right)=-\frac{\beta}{\alpha}-\frac{k}{2}, \quad-\frac{\beta}{\alpha}-\frac{k}{2}<f_{0}\left(-\frac{\beta}{\alpha}+\frac{k}{2}\right)<-\frac{\beta}{\alpha} .
\end{gathered}
$$

Кроме того, при $\alpha k>\sqrt{2 \pi}$ определим числа $x_{1}$ и $x_{2}$ как максимальные корни уравнениц $f_{0}^{\prime}\left(x_{1}\right)=0$ и $f_{0}\left(x_{2}\right)=-\frac{\beta}{\alpha}-\frac{k}{2}$ соответственно. Тогда

$$
-\frac{\beta}{\alpha}<x_{1}<x_{2}<-\frac{\beta}{\alpha}+\frac{k}{2}
$$

причем

$$
x_{1}=-\frac{\beta}{\alpha}+\frac{1}{\alpha} \sqrt{2 \ln \frac{\alpha k}{\sqrt{2 \pi}}}, \quad f_{0}\left(x_{1}\right)>-\frac{\beta}{\alpha}-k .
$$

Доказательство. Как уже было замечено, функции $f_{i}(x)$ строго возрастают при $\alpha k \leqslant \sqrt{2 \pi}$ и не монотонны при $\alpha k>\sqrt{2 \pi}$.

В случае $\alpha k \leqslant \sqrt{2 \pi}$ существование единственных $x_{3}, x_{4}$, определенных в условиях леммы, следует из строгого возрастания $f_{0}(x)$. 
Пусть $\alpha k>\sqrt{2 \pi}$; найдем максимальный корень уравнения $f_{0}^{\prime}\left(x_{1}\right)=0$ :

$$
1-\frac{\alpha k}{\sqrt{2 \pi}} e^{-\frac{1}{2}\left(\alpha x_{1}+\beta\right)^{2}}=0
$$

следовательно,

$$
\frac{1}{2}\left(\alpha x_{1}+\beta\right)^{2}=\ln \frac{\alpha k}{\sqrt{2 \pi}},
$$

откуда в силу условия максимальности

$$
x_{1}=-\frac{\beta}{\alpha}+\frac{1}{\alpha} \sqrt{2 \ln \frac{\alpha k}{\sqrt{2 \pi}}}>-\frac{\beta}{\alpha} .
$$

При этом

$$
\begin{aligned}
f_{0}\left(x_{1}\right)=-\frac{\beta}{\alpha}+\frac{1}{\alpha} \sqrt{2 \ln \frac{\alpha k}{\sqrt{2 \pi}}}-k \Phi\left(-\beta+\sqrt{2 \ln \frac{\alpha k}{\sqrt{2 \pi}}}+\beta\right)> \\
>-\frac{\beta}{\alpha}-k \Phi\left(\sqrt{2 \ln \frac{\alpha k}{\sqrt{2 \pi}}}\right)>-\frac{\beta}{\alpha}-k .
\end{aligned}
$$

Так как в этом случае $f_{i}^{\prime}\left(-\frac{\beta}{\alpha}\right)=1-\frac{\alpha k}{\sqrt{2 \pi}}<0$, то функции $f_{i}(x)$ строго убывают от точки $-\frac{\beta}{\alpha}$ до точки $x_{1}$, после чего строго и неограниченно возрастают. Следовательно, существуют единственные определенные в условиях леммы $x_{2}, x_{3}, x_{4}>x_{1}$, а именно такие, что $f_{0}\left(x_{2}\right)=f_{0}\left(-\frac{\beta}{\alpha}\right)=-\frac{\beta}{\alpha}-\frac{k}{2}, f_{0}\left(x_{3}\right)=-\frac{\beta}{\alpha}, f_{0}\left(x_{4}\right)=-\frac{\beta}{\alpha}+\frac{k}{2}$.

Кроме того, так как $f(x)<-\frac{\beta}{\alpha}-\frac{k}{2}$ для любого $x \in\left(-\frac{\beta}{\alpha}, x_{2}\right)$, то из неравенства

$$
f_{0}\left(-\frac{\beta}{\alpha}+\frac{k}{2}\right)=-\frac{\beta}{\alpha}+\frac{k}{2}-k \Phi\left(\frac{\alpha k}{2}\right)>-\frac{\beta}{\alpha}-\frac{k}{2}=f_{0}\left(x_{2}\right)
$$

следует, что $x_{2}<-\frac{\beta}{\alpha}+\frac{k}{2}$.

Получим теперь оценки чисел $x_{3}$ и $x_{4}$ для произвольных $\alpha, k>0$, пользуясь возрастанием $f_{0}(x)$ при $x \geqslant x_{3}$.

Так как

$$
f_{0}\left(-\frac{\beta}{\alpha}+\frac{k}{2}\right)=-\frac{\beta}{\alpha}+\frac{k}{2}-k \Phi\left(\frac{\alpha k}{2}\right) \in\left(-\frac{\beta}{\alpha}-\frac{k}{2},-\frac{\beta}{\alpha}\right),
$$

то $f_{0}\left(-\frac{\beta}{\alpha}+\frac{k}{2}\right)<-\frac{\beta}{\alpha}=f_{0}\left(x_{3}\right)$, поэтому из возрастания $f_{0}(x)$ при $x \geqslant x_{3}$ следует, что $x_{3}>-\frac{\beta}{\alpha}+\frac{k}{2}$, при этом $-\frac{\beta}{\alpha}-\frac{k}{2}<f_{0}\left(-\frac{\beta}{\alpha}+\frac{k}{2}\right)<-\frac{\beta}{\alpha}$.

Аналогично:

$$
f_{0}\left(-\frac{\beta}{\alpha}+k\right)=-\frac{\beta}{\alpha}+k-k \Phi(\alpha k) \in\left(-\frac{\beta}{\alpha},-\frac{\beta}{\alpha}+\frac{k}{2}\right)=\left(f_{0}\left(x_{3}\right), f_{0}\left(x_{4}\right)\right),
$$

откуда $x_{3}<-\frac{\beta}{\alpha}+k<x_{4}$.

Наконец,

$$
f_{0}\left(-\frac{\beta}{\alpha}+\frac{3 k}{2}\right)=-\frac{\beta}{\alpha}+\frac{3 k}{2}-k \Phi\left(\frac{3 \alpha k}{2}\right)>-\frac{\beta}{\alpha}+\frac{3 k}{2}-k=f_{0}\left(x_{4}\right),
$$

поэтому $x_{4}<-\frac{\beta}{\alpha}+\frac{3 k}{2}$. 
Лемма 3. Пусть

$$
u_{t}(x)=1-\frac{x}{\sqrt{2 \pi}} e^{-\frac{1}{2} t^{2} x^{2}}, \quad \widetilde{u}_{3 / 2}(x)=1-\frac{x}{3 \sqrt{2 \pi}} e^{-\frac{9}{8} x^{2}} .
$$

Тогда при $x>0$ u $t \in\left[\frac{1}{2}, \frac{3}{2}\right]$

$$
0<\frac{1}{2}<u_{t}(x)<\widetilde{u}_{3 / 2}(x)<1 .
$$

Доказательство. Неравенство $\widetilde{u}_{3 / 2}(x)<1$ следует из условия $x>0$.

Так как $\frac{1}{2} \leqslant t \leqslant \frac{3}{2}$, то $e^{-\frac{1}{8} x^{2}} \geqslant e^{-\frac{1}{2} t^{2} x^{2}} \geqslant e^{-\frac{9}{8} x^{2}}$, откуда

$$
1-\frac{x}{\sqrt{2 \pi}} e^{-\frac{1}{8} x^{2}} \leqslant 1-\frac{x}{\sqrt{2 \pi}} e^{-\frac{1}{2} t^{2} x^{2}} \leqslant 1-\frac{x}{\sqrt{2 \pi}} e^{-\frac{9}{8} x^{2}}<1-\frac{x}{3 \sqrt{2 \pi}} e^{-\frac{9}{8} x^{2}},
$$

поэтому достаточно доказать, что $u_{1 / 2}(x)=1-\frac{x}{\sqrt{2 \pi}} e^{-\frac{1}{8} x^{2}}>\frac{1}{2}$.

Легко проверить, что

$$
u_{1 / 2}^{\prime}(x)=\frac{1}{\sqrt{2 \pi}} e^{-\frac{1}{8} x^{2}}\left(\frac{x^{2}}{4}-1\right)
$$

и $u_{1 / 2}^{\prime}(2)=0$. Так как при $0<x<2$ выражение в скобках отрицательно, а при $x>2$ положительно, то минимальное значение $u_{1 / 2}(x)$ принимает в точке $x=2$.

Вычислив значение интересующей нас функции в этой точке, имеем: $0.52>$ $u_{1 / 2}(2)>0.51>\frac{1}{2}$, что и требовалось.

Лемма 4. При $x>0 u t \in\left[\frac{1}{2}, \frac{3}{2}\right]$

$$
u_{t}(x) \exp \left\{\frac{x^{2}(1-\Phi(t x))}{3 \widetilde{u}_{3 / 2}(x)}\right\} \leqslant \widetilde{u}_{3 / 2}(x) .
$$

Доказательство. Пользуясь леммой 3, разделим обе части доказываемого неравенства на $u_{t}(x)$, после чего прологарифмируем их.

Затем оценим правую часть снизу с помощью неравенства $\log x \geqslant 1-\frac{1}{x}$ :

$$
\log \frac{\widetilde{u}_{3 / 2}(x)}{u_{t}(x)} \geqslant \frac{\frac{x}{\sqrt{2 \pi}} e^{-\frac{1}{2} t^{2} x^{2}}-\frac{x}{3 \sqrt{2 \pi}} e^{-\frac{9}{8} x^{2}}}{\widetilde{u}_{3 / 2}(x)} .
$$

Снова применяя лемму 3 и умножая обе части доказываемого неравенства на $\widetilde{u}_{3 / 2}(x)$, получаем, что достаточно установить следующее неравенство:

$$
\frac{x^{2}}{3}(1-\Phi(t x)) \leqslant \frac{x}{\sqrt{2 \pi}} e^{-\frac{1}{2} t^{2} x^{2}}-\frac{x}{3 \sqrt{2 \pi}} e^{-\frac{9}{8} x^{2}} .
$$

Воспользуемся известной оценкой для хвоста функции нормального распределения (см., например, [3]):

$$
1-\Phi(t x)=\frac{1}{\sqrt{2 \pi}} \int_{t x}^{+\infty} e^{-\frac{1}{2} v^{2}} \mathrm{~d} v \leqslant \frac{1}{\sqrt{2 \pi}} \int_{t x}^{+\infty} \frac{v}{t x} e^{-\frac{1}{2} v^{2}} \mathrm{~d} v=\frac{1}{\sqrt{2 \pi} t x} e^{-\frac{1}{2} t^{2} x^{2}},
$$

так как $v \geqslant t x>0$.

Тогда требуемое неравенство примет следующий вид:

$$
\frac{x}{3 \sqrt{2 \pi} t} e^{-\frac{1}{2} t^{2} x^{2}} \leqslant \frac{x}{\sqrt{2 \pi}} e^{-\frac{1}{2} t^{2} x^{2}}-\frac{x}{3 \sqrt{2 \pi}} e^{-\frac{9}{8} x^{2}},
$$


что эквивалентно

$$
\frac{x}{3 \sqrt{2 \pi}} e^{-\frac{9}{8} x^{2}} \leqslant \frac{x}{\sqrt{2 \pi}}\left(1-\frac{1}{3 t}\right) e^{-\frac{1}{2} t^{2} x^{2}} .
$$

При $t \geqslant \frac{1}{2}$ выражение в скобках не меньше $\frac{1}{3}$, поэтому правая часть неравенства положительна. Пользуясь тем, что $t \leqslant \frac{3}{2}$, экспоненту в правой части можно оценить снизу: $e^{-\frac{1}{2} t^{2} x^{2}} \geqslant e^{-\frac{9}{8} x^{2}}$. После умножения обеих частей на $3 \sqrt{2 \pi} \frac{t}{x} e^{\frac{9}{8} x^{2}}$ остается доказать неравенство $t \leqslant 3 t-1$, которое следует из условия $t \geqslant \frac{1}{2}$.

Лемма 5. При $x>\sqrt{2 \pi}$

$$
\left(\frac{x}{\sqrt{2 \pi}}-1\right) \exp \left\{-\frac{x^{2}}{3 \widetilde{u}_{3 / 2}(x)}\left(1-\Phi\left(\sqrt{2 \ln \frac{x}{\sqrt{2 \pi}}}\right)\right)\right\}<e^{-1 / 330}<\widetilde{u}_{3 / 2}(x) .
$$

Доказательство. Найдем производную правой части:

$$
\widetilde{u}_{3 / 2}^{\prime}(x)=\frac{1}{\sqrt{2 \pi}} e^{-\frac{9}{8} x^{2}}\left(\frac{3}{4} x^{2}-\frac{1}{3}\right) .
$$

Так как $x>\sqrt{2 \pi}>\frac{2}{3}$, то выражение в скобках положительно, поэтому функция в правой части неравенства возрастает.

Вычислим ее значение в точке $\sqrt{2 \pi}: 0.9997<\widetilde{u}_{3 / 2}(\sqrt{2 \pi})<0.9998$. Так как при этом $0.996<e^{-1 / 330}<0.997$, то вторая часть требуемого утверждения выполняется.

Учитывая, что $\widetilde{u}_{3 / 2}(x)<1$, оценим выражение в левой части следующим образом:

$$
\begin{aligned}
\left(\frac{x}{\sqrt{2 \pi}}-1\right) \exp \left\{-\frac{x^{2}}{3 \widetilde{u}_{3 / 2}(x)}(1-\Phi\right. & \left.\left.\left(\sqrt{2 \ln \frac{x}{\sqrt{2 \pi}}}\right)\right)\right\}< \\
& <\frac{x}{\sqrt{2 \pi}} \exp \left\{-\frac{x^{2}}{3}\left(1-\Phi\left(\sqrt{2 \ln \frac{x}{\sqrt{2 \pi}}}\right)\right)\right\} .
\end{aligned}
$$

Произведем при $y>0$ замену $x=\sqrt{2 \pi} e^{\frac{1}{2} y^{2}}$ и прологарифмируем обе части. В таком случае остается доказать неравенство

$$
\frac{y^{2}}{2}-\frac{2 \pi}{3} e^{y^{2}}(1-\Phi(y))<-\frac{1}{330},
$$

что эквивалентно

$$
v_{1}(y)=1-\Phi(y)-\frac{3 y^{2}}{4 \pi} e^{-y^{2}}-\frac{1}{220 \pi} e^{-y^{2}}>0 .
$$

Заметим, что $\lim _{y \rightarrow+\infty} v_{1}(y)=0$, поэтому для доказательства требуемого утверждения достаточно показать, что производная $v_{1}(y)$ строго отрицательна.

Вычислим эту производную:

$$
\begin{gathered}
v_{1}^{\prime}(y)=-\frac{1}{\sqrt{2 \pi}} e^{-y^{2}}\left(e^{\frac{1}{2} y^{2}}+\frac{3 y}{\sqrt{2 \pi}}-\frac{3 y^{3}}{\sqrt{2 \pi}}-\frac{y}{55 \sqrt{2 \pi}}\right)= \\
=-\frac{1}{\sqrt{2 \pi}} e^{-y^{2}}\left(e^{\frac{1}{2} y^{2}}-\frac{3}{\sqrt{2 \pi}} y\left(y-\sqrt{\frac{164}{165}}\right)\left(y+\sqrt{\frac{164}{165}}\right)\right) .
\end{gathered}
$$

При $0<y \leqslant \sqrt{\frac{164}{165}}$ выражение в скобках строго положительно, поэтому $v_{1}^{\prime}(y)<0$. Таким образом, остается доказать, что

$$
e^{\frac{1}{2} y^{2}}>\frac{3}{\sqrt{2 \pi}} y\left(y-\sqrt{\frac{164}{165}}\right)\left(y+\sqrt{\frac{164}{165}}\right)
$$


при $y>\sqrt{\frac{164}{165}}$. Прологарифмируем обе части неравенства и перенесем их в одну сторону, тогда требуемое утверждение примет вид

$$
v_{2}(y)=\frac{y^{2}}{2}-\log \frac{3}{\sqrt{2 \pi}}-\log y-\log \left(y-\sqrt{\frac{164}{165}}\right)-\log \left(y+\sqrt{\frac{164}{165}}\right)>0 .
$$

Снова найдем производную функции в левой части:

$$
v_{2}^{\prime}(y)=y-\frac{1}{y}-\frac{1}{y-\sqrt{\frac{164}{165}}}-\frac{1}{y+\sqrt{\frac{164}{165}}}=\frac{165 y^{4}-659 y^{2}+164}{165 y^{3}-164 y} .
$$

Решая соответствующее биквадратное уравнение, получаем, что при $y>\sqrt{\frac{164}{165}}$ оно имеет только один корень $y=\sqrt{\frac{41}{11}}$.

Так как предел $v_{2}^{\prime}(y)$ равен $-\infty$ при $y \downarrow \sqrt{\frac{164}{165}}$ и $+\infty$ при $y \rightarrow+\infty$, то функция $v_{2}(y)$ строго убывает на интервале $\left(\sqrt{\frac{164}{165}}, \sqrt{\frac{41}{11}}\right)$, после чего строго возрастает.

Вычислив ее значение в точке $\sqrt{\frac{41}{11}}$, найдем ее минимум: $0.03>v_{2}\left(\sqrt{\frac{41}{11}}\right)>$ $0.02>0$, что и требовалось.

Кроме того, верно следующее утверждение.

Утверждение 2. При $x \geqslant-\frac{\beta}{\alpha}$ функиия $f_{0}^{\prime}(x)$ строго возрастает.

Доказательство. Действительно,

$$
f_{0}^{\prime \prime}(x)=\frac{\alpha^{2} k}{\sqrt{2 \pi}} e^{-\frac{1}{2}(\alpha x+\beta)^{2}}(\alpha x+\beta) \geqslant 0
$$

при $x \geqslant-\frac{\beta}{\alpha}$, причем $f_{0}^{\prime \prime}(x)=0$ только в одной точке $x=-\frac{\beta}{\alpha}$. Следовательно, $f_{0}^{\prime}(x)$ строго возрастает, что и требовалось.

\section{3. Доказательство теоремы 3}

Докажем теперь неравенство

$$
\sup _{x} \Psi_{q}(x) \leqslant r<1
$$

разбив всю область значений $x$ на отдельные полуинтервалы в зависимости от знаков выражений под модулями.

Случай 1. $\alpha k \leqslant \sqrt{2 \pi}, x \in\left[-\frac{\beta}{\alpha},-\frac{\beta}{\alpha}+\frac{k}{2}\right)$.

Доказательство. Из условия $\alpha k \leqslant \sqrt{2 \pi}$ следует, что $f_{0}(x)$ строго возрастает, поэтому по лемме 2

$$
-\frac{\beta}{\alpha}-\frac{k}{2}=f_{0}\left(-\frac{\beta}{\alpha}\right) \leqslant f_{0}(x)<f_{0}\left(-\frac{\beta}{\alpha}+\frac{k}{2}\right)<-\frac{\beta}{\alpha} .
$$


Таким образом,

$$
\begin{aligned}
x \in\left[-\frac{\beta}{\alpha},-\frac{\beta}{\alpha}+\frac{k}{2}\right) & \Longrightarrow \lambda(x)=-x-\frac{\beta}{\alpha}+\frac{k}{2}, \\
f_{0}(x) \in\left[-\frac{\beta}{\alpha}-\frac{k}{2},-\frac{\beta}{\alpha}\right) & \Longrightarrow \lambda\left(f_{0}(x)\right)=f_{0}(x)+\frac{\beta}{\alpha}+\frac{k}{2}, \\
f_{1}(x) \in\left[-\frac{\beta}{\alpha}+\frac{k}{2},-\frac{\beta}{\alpha}+k\right) & \Longrightarrow \lambda\left(f_{1}(x)\right)=f_{1}(x)+\frac{\beta}{\alpha}-\frac{k}{2}=f_{0}(x)+\frac{\beta}{\alpha}+\frac{k}{2} .
\end{aligned}
$$

Так как $f_{i}(x)$ возрастают, то $L_{x}(f)=f_{i}^{\prime}(x)$ и

$$
\begin{gathered}
\Psi_{q}(x)=L_{x}(f) \frac{\exp \left\{c\left(f_{0}(x)+\frac{\beta}{\alpha}+\frac{k}{2}\right)\right\}}{\exp \left\{c\left(-x-\frac{\beta}{\alpha}+\frac{k}{2}\right)\right\}}= \\
\quad=\left(1-\frac{\alpha k}{\sqrt{2 \pi}} e^{-\frac{1}{2}(\alpha x+\beta)^{2}}\right) \exp \left\{c\left(x+f_{0}(x)+\frac{2 \beta}{\alpha}\right)\right\} .
\end{gathered}
$$

Поскольку $f_{0}(x)$ возрастает по условию, а $f_{0}^{\prime}(x)$ по утверждению 2 , то возрастает также и $\Psi_{q}(x)$. Следовательно,

$$
\Psi_{q}(x)<\Psi_{q}\left(-\frac{\beta}{\alpha}+\frac{k}{2}\right)=\left(1-\frac{\alpha k}{\sqrt{2 \pi}} e^{-\frac{\alpha^{2} k^{2}}{8}}\right) \exp \left\{c k\left(1-\Phi\left(\frac{\alpha k}{2}\right)\right)\right\} .
$$

Заметим, что по определению

$$
c k=\frac{\alpha^{2} k^{2}}{3 \widetilde{u}_{3 / 2}(\alpha k)} .
$$

Применяя лемму 4 при $x=\alpha k$ и $t=\frac{1}{2}$, получаем, что

$$
\Psi_{q}(x)<u_{1 / 2}(\alpha k) \exp \left\{\frac{\alpha^{2} k^{2}}{3 \widetilde{u}_{3 / 2}(\alpha k)}\left(1-\Phi\left(\frac{\alpha k}{2}\right)\right)\right\} \leqslant \widetilde{u}_{3 / 2}(\alpha k) \leqslant r .
$$

Случай 2. $\alpha k>\sqrt{2 \pi}, x \in\left[-\frac{\beta}{\alpha}, x_{1}\right)$.

Доказательство. Это единственный случай, когда $f_{0}(x)$ строго убывает. По лемме 2

$$
-\frac{\beta}{\alpha}-\frac{k}{2}=f_{0}\left(-\frac{\beta}{\alpha}\right) \geqslant f_{0}(x)>f_{0}\left(x_{1}\right)>-\frac{\beta}{\alpha}-k .
$$

Таким образом,

$$
\begin{gathered}
x \in\left[-\frac{\beta}{\alpha},-\frac{\beta}{\alpha}+\frac{k}{2}\right) \Longrightarrow \lambda(x)=-x-\frac{\beta}{\alpha}+\frac{k}{2}, \\
f_{0}(x) \in\left(-\frac{\beta}{\alpha}-k,-\frac{\beta}{\alpha}-\frac{k}{2}\right] \Longrightarrow \lambda\left(f_{0}(x)\right)=-f_{0}(x)-\frac{\beta}{\alpha}-\frac{k}{2}, \\
f_{1}(x) \in\left(-\frac{\beta}{\alpha},-\frac{\beta}{\alpha}+\frac{k}{2}\right] \Longrightarrow \lambda\left(f_{1}(x)\right)=-f_{1}(x)-\frac{\beta}{\alpha}+\frac{k}{2}=-f_{0}(x)-\frac{\beta}{\alpha}-\frac{k}{2} .
\end{gathered}
$$

Так как функции $f_{i}(x)$ убывают, то

$$
L_{x}(f)=-f_{i}^{\prime}(x)=-1+\frac{\alpha k}{\sqrt{2 \pi}} e^{-\frac{1}{2}(\alpha x+\beta)^{2}} \leqslant \frac{\alpha k}{\sqrt{2 \pi}}-1 .
$$


Отсюда

$$
\begin{aligned}
& \Psi_{q}(x)=L_{x}(f) \frac{\exp \left\{c\left(-f_{0}(x)-\frac{\beta}{\alpha}-\frac{k}{2}\right)\right\}}{\exp \left\{c\left(-x-\frac{\beta}{\alpha}+\frac{k}{2}\right)\right\}} \\
& \leqslant\left(\frac{\alpha k}{\sqrt{2 \pi}}-1\right) \exp \left\{c\left(x-f_{0}(x)-k\right)\right\}= \\
&=\left(\frac{\alpha k}{\sqrt{2 \pi}}-1\right) \exp \{c k(\Phi(\alpha x+\beta)-1)\} .
\end{aligned}
$$

По определению $x_{1}$

$$
\alpha x+\beta<\alpha x_{1}+\beta=\sqrt{2 \ln \frac{\alpha k}{\sqrt{2 \pi}}} .
$$

Следовательно,

$$
\Psi_{q}(x)<\left(\frac{\alpha k}{\sqrt{2 \pi}}-1\right) \exp \left\{-c k\left(1-\Phi\left(\sqrt{2 \ln \frac{\alpha k}{\sqrt{2 \pi}}}\right)\right)\right\} .
$$

Пользуясь выражением (3) и леммой 5 при $x=\alpha k$, получаем:

$$
\Psi_{q}(x)<\left(\frac{\alpha k}{\sqrt{2 \pi}}-1\right) \exp \left\{-\frac{\alpha^{2} k^{2}}{3 \widetilde{u}_{3 / 2}(x)}\left(1-\Phi\left(\sqrt{2 \ln \frac{\alpha k}{\sqrt{2 \pi}}}\right)\right)\right\}<\widetilde{u}_{3 / 2}(\alpha k) \leqslant r
$$

Случай 3. $\alpha k>\sqrt{2 \pi}, x \in\left[x_{1}, x_{2}\right)$.

Доказательство. По лемме 2

$$
-\frac{\beta}{\alpha}-k<f_{0}\left(x_{1}\right) \leqslant f_{0}(x)<f_{0}\left(x_{2}\right)=-\frac{\beta}{\alpha}-\frac{k}{2} .
$$

Таким образом,

$$
\begin{gathered}
x \in\left(-\frac{\beta}{\alpha},-\frac{\beta}{\alpha}+\frac{k}{2}\right) \Longrightarrow \lambda(x)=-x-\frac{\beta}{\alpha}+\frac{k}{2}, \\
f_{0}(x) \in\left(-\frac{\beta}{\alpha}-k,-\frac{\beta}{\alpha}-\frac{k}{2}\right) \Longrightarrow \lambda\left(f_{0}(x)\right)=-f_{0}(x)-\frac{\beta}{\alpha}-\frac{k}{2}, \\
f_{1}(x) \in\left(-\frac{\beta}{\alpha},-\frac{\beta}{\alpha}+\frac{k}{2}\right) \Longrightarrow \lambda\left(f_{1}(x)\right)=-f_{1}(x)-\frac{\beta}{\alpha}+\frac{k}{2}=-f_{0}(x)-\frac{\beta}{\alpha}-\frac{k}{2} .
\end{gathered}
$$

Так как $L_{x}(f)=f_{i}^{\prime}(x)$ и $f_{0}^{\prime}(x)$ возрастает по утверждению 2 , то

$$
L_{x}(f)<L_{-\frac{\beta}{\alpha}+\frac{k}{2}}(f)=1-\frac{\alpha k}{\sqrt{2 \pi}} e^{-\frac{\alpha^{2} k^{2}}{8}}=u_{1 / 2}(\alpha k) .
$$

Следовательно,

$$
\begin{aligned}
\Psi_{q}(x)=L_{x}(f) \frac{\exp \left\{c\left(-f_{0}(x)-\frac{\beta}{\alpha}-\frac{k}{2}\right)\right\}}{\exp \left\{c\left(-x-\frac{\beta}{\alpha}+\frac{k}{2}\right)\right\}}< & \\
\quad<u_{1 / 2}(\alpha k) \exp \{c(x- & \left.\left.f_{0}(x)-k\right)\right\}= \\
& =u_{1 / 2}(\alpha k) \exp \{c k(\Phi(\alpha x+\beta)-1)\} .
\end{aligned}
$$


В силу того, что $x<-\frac{\beta}{\alpha}+\frac{k}{2}$, получаем оценку

$$
\Psi_{q}(x)<u_{1 / 2}(\alpha k) \exp \left\{-c k\left(1-\Phi\left(\frac{\alpha k}{2}\right)\right)\right\} \text {. }
$$

Заметим теперь, что из выражения (3) и леммы 3 следует, что $c k>0$, откуда по той же лемме 3

$$
\Psi_{q}(x)<u_{1 / 2}(\alpha k)<\widetilde{u}_{3 / 2}(\alpha k) \leqslant r .
$$

Случай 4. $\alpha k>\sqrt{2 \pi}, x \in\left[x_{2},-\frac{\beta}{\alpha}+\frac{k}{2}\right)$.

Доказательство. По лемме 2

$$
-\frac{\beta}{\alpha}-\frac{k}{2}=f_{0}\left(x_{2}\right) \leqslant f_{0}(x)<f_{0}\left(-\frac{\beta}{\alpha}+\frac{k}{2}\right)<-\frac{\beta}{\alpha} .
$$

Таким образом,

$$
\begin{aligned}
& x \in\left(-\frac{\beta}{\alpha},-\frac{\beta}{\alpha}+\frac{k}{2}\right) \Longrightarrow \lambda(x)=-x-\frac{\beta}{\alpha}+\frac{k}{2}, \\
& f_{0}(x) \in\left[-\frac{\beta}{\alpha}-\frac{k}{2},-\frac{\beta}{\alpha}\right) \Longrightarrow \lambda\left(f_{0}(x)\right)=f_{0}(x)+\frac{\beta}{\alpha}+\frac{k}{2}, \\
& f_{1}(x) \in\left[-\frac{\beta}{\alpha}+\frac{k}{2},-\frac{\beta}{\alpha}+k\right) \Longrightarrow \lambda\left(f_{1}(x)\right)=f_{1}(x)+\frac{\beta}{\alpha}-\frac{k}{2}=f_{0}(x)+\frac{\beta}{\alpha}+\frac{k}{2} .
\end{aligned}
$$

Как и в предыдущем случае $3, L_{x}(f)<u_{1 / 2}(\alpha k)$, поэтому

$$
\begin{aligned}
& \Psi_{q}(x)=L_{x}(f) \frac{\exp \left\{c\left(f_{0}(x)+\frac{\beta}{\alpha}+\frac{k}{2}\right)\right\}}{\exp \left\{c\left(-x-\frac{\beta}{\alpha}+\frac{k}{2}\right)\right\}}< \\
& <u_{1 / 2}(\alpha k) \exp \left\{c\left(x+f_{0}(x)+\frac{2 \beta}{\alpha}\right)\right\}= \\
& =u_{1 / 2}(\alpha k) \exp \left\{c\left(2 x-k \Phi(\alpha x+\beta)+\frac{2 \beta}{\alpha}\right)\right\} .
\end{aligned}
$$

В силу того, что $x<-\frac{\beta}{\alpha}+\frac{k}{2}$, имеем оценку

$$
\Psi_{q}(x)<u_{1 / 2}(\alpha k) \exp \left\{c k\left(1-\Phi\left(\frac{\alpha k}{2}\right)\right)\right\},
$$

совпадающую с оценкой в случае 1. Следовательно,

$$
\Psi_{q}(x)<\widetilde{u}_{3 / 2}(\alpha k) \leqslant r .
$$

Случай 5. $x \in\left[-\frac{\beta}{\alpha}+\frac{k}{2}, x_{3}\right)$.

Доказательство. По лемме 2

$$
-\frac{\beta}{\alpha}-\frac{k}{2}<f_{0}\left(-\frac{\beta}{\alpha}+\frac{k}{2}\right) \leqslant f_{0}(x)<f_{0}\left(x_{3}\right)=-\frac{\beta}{\alpha} .
$$


Таким образом,

$$
\begin{aligned}
x \in\left[-\frac{\beta}{\alpha}+\frac{k}{2},-\frac{\beta}{\alpha}+k\right) & \Longrightarrow \lambda(x)=x+\frac{\beta}{\alpha}-\frac{k}{2}, \\
f_{0}(x) \in\left(-\frac{\beta}{\alpha}-\frac{k}{2},-\frac{\beta}{\alpha}\right) & \Longrightarrow \lambda\left(f_{0}(x)\right)=f_{0}(x)+\frac{\beta}{\alpha}+\frac{k}{2}, \\
f_{1}(x) \in\left(-\frac{\beta}{\alpha}+\frac{k}{2},-\frac{\beta}{\alpha}+k\right) & \Longrightarrow \lambda\left(f_{1}(x)\right)=f_{1}(x)+\frac{\beta}{\alpha}-\frac{k}{2}=f_{0}(x)+\frac{\beta}{\alpha}+\frac{k}{2} .
\end{aligned}
$$

Так как $f_{i}(x)$ возрастают, то $L_{x}(f)=f_{i}^{\prime}(x)$ и

$$
\Psi_{q}(x)=L_{x}(f) \frac{\exp \left\{c\left(f_{0}(x)+\frac{\beta}{\alpha}+\frac{k}{2}\right)\right\}}{\exp \left\{c\left(x+\frac{\beta}{\alpha}-\frac{k}{2}\right)\right\}}=f_{0}^{\prime}(x) \exp \left\{c\left(-x+f_{0}(x)+k\right)\right\} .
$$

Докажем, что функция $\Psi_{q}(x)$ возрастает.

Производная второго множителя в выражении для $\Psi_{q}(x)$ равняется

$$
c\left(f_{0}^{\prime}(x)-1\right) \exp \left\{c\left(-x+f_{0}(x)+k\right)\right\} .
$$

Следовательно,

$$
\begin{aligned}
\Psi_{q}^{\prime}(x)=f_{0}^{\prime \prime}(x) \exp \left\{c\left(-x+f_{0}(x)+k\right)\right\} & + \\
& +f_{0}^{\prime}(x) c\left(f_{0}^{\prime}(x)-1\right) \exp \left\{c\left(-x+f_{0}(x)+k\right)\right\} .
\end{aligned}
$$

Заметим, используя выражение (2), что верно представление

$$
f_{0}^{\prime \prime}(x)=-\alpha\left(f_{0}^{\prime}(x)-1\right)(\alpha x+\beta),
$$

откуда

$$
\Psi_{q}^{\prime}(x)=-\left(f_{0}^{\prime}(x)-1\right)\left(\alpha(\alpha x+\beta)-c f_{0}^{\prime}(x)\right) \exp \left\{c\left(-x+f_{0}(x)+k\right)\right\} .
$$

В силу оценки $-\frac{\beta}{\alpha}+\frac{k}{2} \leqslant x<-\frac{\beta}{\alpha}+k$ и убывания функции $-f_{0}^{\prime}(x)$ по утверждению 2, имеем следующее неравенство:

$$
\alpha(\alpha x+\beta)-c f_{0}^{\prime}(x) \geqslant \frac{\alpha^{2} k}{2}-c\left(1-\frac{\alpha k}{\sqrt{2 \pi}} e^{-\frac{1}{2} \alpha^{2} k^{2}}\right) .
$$

Учитывая определение $u_{t}(x)$, выражение (3) и лемму 3 при $x=\alpha k$ и $t=1$, получаем:

$$
\frac{\alpha^{2} k}{2}-c\left(1-\frac{\alpha k}{\sqrt{2 \pi}} e^{-\frac{1}{2} \alpha^{2} k^{2}}\right)=\frac{\alpha^{2} k}{2}-\frac{\alpha^{2} k}{3 \widetilde{u}_{3 / 2}(\alpha k)} u_{1}(\alpha k)>\frac{\alpha^{2} k}{2}-\frac{\alpha^{2} k}{3}=\frac{\alpha^{2} k}{6}>0 .
$$

Наконец, так как

$$
-\left(f_{0}^{\prime}(x)-1\right)=\frac{\alpha k}{\sqrt{2 \pi}} e^{-\frac{1}{2}(\alpha x+\beta)^{2}}>0,
$$

окончательно получаем, что $\Psi_{q}^{\prime}(x)>0$ и функция $\Psi_{q}(x)$ возрастает, поэтому

$$
\Psi_{q}(x)<\Psi_{q}\left(-\frac{\beta}{\alpha}+k\right)=\left(1-\frac{\alpha k}{\sqrt{2 \pi}} e^{-\frac{\alpha^{2} k^{2}}{2}}\right) \exp \{c k(1-\Phi(\alpha k))\}
$$


Применяя лемму 4 при $x=\alpha k$ и $t=1$, получаем, что

$$
\Psi_{q}(x)<u_{1}(\alpha k) \exp \left\{\frac{\alpha^{2} k^{2}}{3 \widetilde{u}_{3 / 2}(\alpha k)}(1-\Phi(\alpha k))\right\} \leqslant \widetilde{u}_{3 / 2}(\alpha k) \leqslant r .
$$

Случай 6. $x \in\left[x_{3}, x_{4}\right)$.

Доказательство. По лемме 2

$$
-\frac{\beta}{\alpha}=f_{0}\left(x_{3}\right) \leqslant f_{0}(x)<f_{0}\left(x_{4}\right)=-\frac{\beta}{\alpha}+\frac{k}{2} .
$$

Таким образом,

$$
\begin{aligned}
& x \in\left(-\frac{\beta}{\alpha}+\frac{k}{2},-\frac{\beta}{\alpha}+\frac{3 k}{2}\right) \Longrightarrow \lambda(x)=x+\frac{\beta}{\alpha}-\frac{k}{2}, \\
& f_{0}(x) \in\left[-\frac{\beta}{\alpha},-\frac{\beta}{\alpha}+\frac{k}{2}\right) \Longrightarrow \lambda\left(f_{0}(x)\right)=-f_{0}(x)-\frac{\beta}{\alpha}+\frac{k}{2}, \\
& f_{1}(x) \in\left[-\frac{\beta}{\alpha}+k,-\frac{\beta}{\alpha}+\frac{3 k}{2}\right) \Longrightarrow \lambda\left(f_{1}(x)\right)=f_{1}(x)+\frac{\beta}{\alpha}-\frac{k}{2}=f_{0}(x)+\frac{\beta}{\alpha}+\frac{k}{2} .
\end{aligned}
$$

Так как $f_{i}(x)$ возрастают, то $L_{x}(f)=f_{i}^{\prime}(x)$, поэтому

$$
\begin{aligned}
& \Psi_{q}(x)=(1-q) f_{0}^{\prime}(x) \frac{\exp \left\{c\left(-f_{0}(x)-\frac{\beta}{\alpha}+\frac{k}{2}\right)\right\}}{\exp \left\{c\left(x+\frac{\beta}{\alpha}-\frac{k}{2}\right)\right\}}+q f_{0}^{\prime}(x) \frac{\exp \left\{c\left(f_{0}(x)+\frac{\beta}{\alpha}+\frac{k}{2}\right)\right\}}{\exp \left\{c\left(x+\frac{\beta}{\alpha}-\frac{k}{2}\right)\right\}}= \\
& =(1-q) f_{0}^{\prime}(x) \exp \left\{c\left(-x-f_{0}(x)-\frac{2 \beta}{\alpha}+k\right)\right\}+q f_{0}^{\prime}(x) \exp \left\{c\left(-x+f_{0}(x)+k\right)\right\} .
\end{aligned}
$$

Докажем, что вторая экспонента не меньше первой, для этого рассмотрим их отношение:

$$
v_{3}(x)=\exp \left\{2 c\left(f_{0}(x)+\frac{\beta}{\alpha}\right)\right\} .
$$

Производная этого отношения

$$
v_{3}^{\prime}(x)=2 c f_{0}^{\prime}(x) \exp \left\{2 c\left(f_{0}(x)+\frac{\beta}{\alpha}\right)\right\}
$$

положительна в силу того, что $c>0$ и $f_{0}^{\prime}(x)>0$ при $x>x_{1}$. Кроме того, на левой границе рассматриваемого полуинтервала $f_{0}\left(x_{3}\right)=-\frac{\beta}{\alpha}$, поэтому $v_{3}\left(x_{3}\right)=1$. Значит, первая экспонента не больше второй.

Следовательно,

$$
\Psi_{q}(x) \leqslant f_{0}^{\prime}(x) \exp \left\{c\left(-x+f_{0}(x)+k\right)\right\} .
$$

Согласно доказательству случая 5, эта функция возрастает при

$$
\alpha(\alpha x+\beta)-c f_{0}^{\prime}(x)>0 .
$$

Учитывая, что $-\frac{\beta}{\alpha}+\frac{k}{2}<x<-\frac{\beta}{\alpha}+\frac{3 k}{2}$ и $-f_{0}^{\prime}(x)$ убывает по утверждению 2 , имеем следующее неравенство:

$$
\alpha(\alpha x+\beta)-c f_{0}^{\prime}(x) \geqslant \frac{\alpha^{2} k}{2}-c\left(1-\frac{\alpha k}{\sqrt{2 \pi}} e^{-\frac{9}{8} \alpha^{2} k^{2}}\right) .
$$


Пользуясь выражением (3) и леммой 3 при $x=\alpha k$ и $t=\frac{3}{2}$, получаем:

$$
\frac{\alpha^{2} k}{2}-c\left(1-\frac{\alpha k}{\sqrt{2 \pi}} e^{-\frac{9}{8} \alpha^{2} k^{2}}\right)=\frac{\alpha^{2} k}{2}-\frac{\alpha^{2} k}{3 \widetilde{u}_{3 / 2}(\alpha k)} u_{3 / 2}(\alpha k)>\frac{\alpha^{2} k}{2}-\frac{\alpha^{2} k}{3}=\frac{\alpha^{2} k}{6}>0,
$$

что и требовалось. Следовательно,

$$
\Psi_{q}(x)<\left(1-\frac{\alpha k}{\sqrt{2 \pi}} e^{-\frac{9}{8} \alpha^{2} k^{2}}\right) \exp \left\{c k\left(1-\Phi\left(\frac{3 \alpha k}{2}\right)\right)\right\} .
$$

Применяя лемму 4 при $x=\alpha k$ и $t=\frac{3}{2}$, находим

$$
\Psi_{q}(x)<u_{3 / 2}(\alpha k) \exp \left\{\frac{\alpha^{2} k^{2}}{3 \widetilde{u}_{3 / 2}(\alpha k)}\left(1-\Phi\left(\frac{3 \alpha k}{2}\right)\right)\right\} \leqslant \widetilde{u}_{3 / 2}(\alpha k) \leqslant r .
$$

Случай 7. $x \in\left[x_{4},+\infty\right)$.

Доказательство. По лемме 2

$$
\begin{aligned}
x \in\left(-\frac{\beta}{\alpha}+k,+\infty\right) & \Longrightarrow \lambda(x)=x+\frac{\beta}{\alpha}-\frac{k}{2} \\
f_{0}(x) \in\left[-\frac{\beta}{\alpha}+\frac{k}{2},+\infty\right) & \Longrightarrow \lambda\left(f_{0}(x)\right)=f_{0}(x)+\frac{\beta}{\alpha}-\frac{k}{2}, \\
f_{1}(x) \in\left[-\frac{\beta}{\alpha}+\frac{3 k}{2},+\infty\right) & \Longrightarrow \lambda\left(f_{1}(x)\right)=f_{1}(x)+\frac{\beta}{\alpha}-\frac{k}{2}=f_{0}(x)+\frac{\beta}{\alpha}+\frac{k}{2} .
\end{aligned}
$$

Так как $f_{i}(x)$ возрастают, то $L_{x}(f)=f_{i}^{\prime}(x)$ и

$$
\begin{gathered}
\Psi_{q}(x)=(1-q) f_{0}^{\prime}(x) \frac{\exp \left\{c\left(f_{0}(x)+\frac{\beta}{\alpha}-\frac{k}{2}\right)\right\}}{\exp \left\{c\left(x+\frac{\beta}{\alpha}-\frac{k}{2}\right)\right\}}+q f_{0}^{\prime}(x) \frac{\exp \left\{c\left(f_{0}(x)+\frac{\beta}{\alpha}+\frac{k}{2}\right)\right\}}{\exp \left\{c\left(x+\frac{\beta}{\alpha}-\frac{k}{2}\right)\right\}}= \\
=(1-q) f_{0}^{\prime}(x) \exp \left\{c\left(-x+f_{0}(x)\right)\right\}+q f_{0}^{\prime}(x) \exp \left\{c\left(-x+f_{0}(x)+k\right)\right\} .
\end{gathered}
$$

Производная $\Psi_{q}^{\prime}(x)$ равна

$$
\begin{aligned}
& \Psi_{q}^{\prime}(x)=(1-q) f_{0}^{\prime \prime}(x) \exp \left\{c\left(-x+f_{0}(x)\right)\right\}+ \\
& +(1-q) f_{0}^{\prime}(x) c\left(f_{0}^{\prime}(x)-1\right) \exp \left\{c\left(-x+f_{0}(x)\right)\right\}+ \\
& +q f_{0}^{\prime \prime}(x) \exp \left\{c\left(-x+f_{0}(x)+k\right)\right\}+ \\
& \quad+q f_{0}^{\prime}(x) c\left(f_{0}^{\prime}(x)-1\right) \exp \left\{c\left(-x+f_{0}(x)+k\right)\right\} .
\end{aligned}
$$

Пользуясь представлением (4), получаем следующее равенство:

$$
\begin{aligned}
& \Psi_{q}^{\prime}(x)=-(1-q) \alpha\left(f_{0}^{\prime}(x)-1\right)(\alpha x+\beta) \exp \left\{c\left(-x+f_{0}(x)\right)\right\}+ \\
& +(1-q) f_{0}^{\prime}(x) c\left(f_{0}^{\prime}(x)-1\right) \exp \left\{c\left(-x+f_{0}(x)\right)\right\}- \\
& -q \alpha\left(f_{0}^{\prime}(x)-1\right)(\alpha x+\beta) \exp \left\{c\left(-x+f_{0}(x)+k\right)\right\}+ \\
& \quad+q f_{0}^{\prime}(x) c\left(f_{0}^{\prime}(x)-1\right) \exp \left\{c\left(-x+f_{0}(x)+k\right)\right\} .
\end{aligned}
$$

Группируя общие члены, имеем:

$$
\begin{aligned}
& \Psi_{q}^{\prime}(x)=-\left(f_{0}^{\prime}(x)-1\right)\left(\alpha(\alpha x+\beta)-c f_{0}^{\prime}(x)\right) \times \\
& \times\left((1-q) \exp \left\{c\left(-x+f_{0}(x)\right)\right\}+q \exp \left\{c\left(-x+f_{0}(x)+k\right)\right\}\right) .
\end{aligned}
$$


Заметим, что из выражения (3) и леммы 3 при $x=\alpha k$ и $t=\frac{3}{2}$ следует, что $\frac{1}{3} \alpha^{2} k<c<\frac{2}{3} \alpha^{2} k$.

Так как $x>-\frac{\beta}{\alpha}+k$ и $f_{0}^{\prime}(x)<1$, то

$$
\alpha(\alpha x+\beta)-c f_{0}^{\prime}(x)>\alpha^{2} k-c>\frac{\alpha^{2} k}{3}>0 .
$$

Отсюда, учитывая (5), получаем, что $\Psi_{q}^{\prime}(x)>0$ и функция $\Psi_{q}(x)$ возрастает. Следовательно,

$$
\Psi_{q}(x) \leqslant \lim _{x \rightarrow+\infty} \Psi_{q}(x)=(1-q) e^{-c k}+q<(1-q) e^{-\frac{1}{3} \alpha^{2} k^{2}}+q \leqslant r .
$$

Окончательно получаем, что

$$
\sup _{x} \Psi_{q}(x) \leqslant r
$$

где

$$
r=\max \left\{1-\frac{\alpha k}{3 \sqrt{2 \pi}} e^{-\frac{9}{8} \alpha^{2} k^{2}},(1-q) e^{-\frac{1}{3} \alpha^{2} k^{2}}+q\right\}<1
$$

в силу того, что $e^{-\frac{1}{3} \alpha^{2} k^{2}}<1$ и $\widetilde{u}_{3 / 2}(\alpha k)<1$ по лемме 3 .

\section{4. Стационарное распределение}

Докажем теперь теорему из статьи [5], связывающую выполнение условия (1) с локальной сжимаемостью итерационной системы.

Теорема 4. Если для непрерывной функции $\psi(x): \mathbb{R} \rightarrow[1,+\infty)$ выполнено условие

$$
\sup _{x} \mathbf{E}\left\{\frac{\psi(f(x))}{\psi(x)} L_{x}(f)\right\} \leqslant r<1,
$$

то итерационная функциональная система $\mathcal{R}$ является локально сжимающей с функиией нормировки $\psi(x)$.

Доказательство. Необходимо доказать, что

$$
U_{n}(x)=\mathbf{E}\left\{L_{x}\left(\widetilde{R}_{n}\right)\right\} \leqslant \psi(x) r^{n}
$$

для всех $x \in \mathbb{R}$ и $n \in \mathbb{N}_{0}$.

Докажем это утверждение индукцией по $n$. База индукции при $n=0$ следует из того, что $\widetilde{R}_{0}(x)=x, U_{0}(x)=\mathbf{E}\left\{L_{x}\left(\widetilde{R}_{0}\right)\right\}=1$ и $\psi(x) \geqslant 1$.

Перейдем к шагу индукции. По утверждению 1

$$
U_{n+1}(x)=\mathbf{E}\left\{L_{x}\left(\widetilde{R}_{n+1}\right)\right\}=\mathbf{E}\left\{L_{x}\left(\widetilde{R}_{n} \circ f_{W_{n+1}}\right)\right\}=\mathbf{E}\left\{L_{f_{W_{n+1}}(x)}\left(\widetilde{R}_{n}\right) L_{x}\left(f_{W_{n+1}}\right)\right\} .
$$

В силу того, что все $W_{i}$ независимы, $\widetilde{R}_{n}$ и $f_{W_{n+1}}$ также независимы, поэтому

$$
U_{n+1}(x)=\mathbf{E}\left\{U_{n}\left(f_{W_{n+1}}(x)\right) L_{x}\left(f_{W_{n+1}}\right)\right\} .
$$

Пользуясь предположением индукции, получаем, что

$$
U_{n+1}(x) \leqslant r^{n} \mathbf{E}\left\{\psi\left(f_{W_{n+1}}(x)\right) L_{x}\left(f_{W_{n+1}}\right)\right\} .
$$


Так как все $W_{i}$ одинаково распределены, то

$$
U_{n+1}(x) \leqslant r^{n} \mathbf{E}\left\{\psi(f(x)) L_{x}(f)\right\}=r^{n} \psi(x) \mathbf{E}\left\{\frac{\psi(f(x))}{\psi(x)} L_{x}(f)\right\} .
$$

По условию теоремы данное математическое ожидание не превосходит $r$, откуда $U_{n+1}(x) \leqslant \psi(x) r^{n+1}$, что и требовалось доказать.

Таким образом, из теорем 3 и 4 следует, что итерационная функциональная система $\mathcal{R}$ является локально сжимающей с функцией нормировки $\psi(x)$.

Теперь можно доказать основную теорему о стационарном распределении $\left\{R_{n}(x)\right\}$.

Доказательство теоремы 2. Воспользуемся теоремой 1, то есть докажем, что предел $\widetilde{R}_{\infty}(x)=\lim _{n \rightarrow+\infty} \widetilde{R}_{n}(x)$ существует почти наверное, конечен и не зависит от $x$.

Рассмотрим некоторое $l \in \mathbb{N}$. По формуле Ньютона-Лейбница

$$
\begin{aligned}
\mathbf{E}\left\{\left|\widetilde{R}_{l}(x)-\widetilde{R}_{l+1}(x)\right|\right\}=\mathbf{E}\{\mid & \left.\widetilde{R}_{l}(x)-\widetilde{R}_{l} \circ f_{W_{l+1}}(x) \mid\right\} \leqslant \\
& \leqslant \mathbf{E}\left\{\left|x-f_{W_{l+1}}(x)\right| \int_{0}^{1} L_{x+t\left(f_{W_{l+1}}(x)-x\right)}\left(\widetilde{R}_{l}\right) \mathrm{d} t\right\} .
\end{aligned}
$$

Оценивая интеграл произведением длины отрезка интегрирования и максимума подынтегральной функции, получаем:

$$
\mathbf{E}\left\{\left|\widetilde{R}_{l}(x)-\widetilde{R}_{l+1}(x)\right|\right\} \leqslant \mathbf{E}\left\{\left|x-f_{W_{l+1}}(x)\right| \sup _{0 \leqslant t \leqslant 1} L_{x+t\left(f_{W_{l+1}}(x)-x\right)}\left(\widetilde{R}_{l}\right)\right\} .
$$

Из независимости $\widetilde{R}_{l}$ и $f_{W_{l+1}}$, а также одинаковой распределенности всех $W_{i}$ имеем:

$$
\mathbf{E}\left\{\left|\widetilde{R}_{l}(x)-\widetilde{R}_{l+1}(x)\right|\right\} \leqslant \mathbf{E}\left\{|x-f(x)| \sup _{0 \leqslant t \leqslant 1} U_{l}(x+t(f(x)-x))\right\} .
$$

По теореме 4 итерационная система $\mathcal{R}$ является локально сжимающей с функцией нормировки $\psi(x)$, поэтому

$$
\mathbf{E}\left\{\left|\widetilde{R}_{l}(x)-\widetilde{R}_{l+1}(x)\right|\right\} \leqslant r^{l} \mathbf{E}\left\{|x-f(x)| \sup _{0 \leqslant t \leqslant 1} \psi(x+t(f(x)-x))\right\} .
$$

Для оценки входящего в эту формулу супремума представим его в следующем виде:

$$
\sup _{0 \leqslant t \leqslant 1} \psi\left(x+t\left(f_{i}(x)-x\right)\right)=\exp \left\{c \sup _{0 \leqslant t \leqslant 1} \lambda\left(x+t\left(f_{i}(x)-x\right)\right)\right\} .
$$

Тогда при $0 \leqslant t \leqslant 1$ верно неравенство

$$
\begin{aligned}
\lambda\left(x+t\left(f_{0}(x)-x\right)\right)=|| x-t k \Phi & (\alpha x+\beta)+\frac{\beta}{\alpha}\left|-\frac{k}{2}\right| \leqslant \\
& \leqslant\left|x+\frac{\beta}{\alpha}\right|+t k \Phi(\alpha x+\beta)+\frac{k}{2} \leqslant\left|x+\frac{\beta}{\alpha}\right|+t k+\frac{k}{2},
\end{aligned}
$$


и аналогично

$$
\begin{aligned}
& \lambda\left(x+t\left(f_{1}(x)-x\right)\right)=|| x+t k(1-\Phi(\alpha x+\beta))+\frac{\beta}{\alpha}\left|-\frac{k}{2}\right| \leqslant \\
& \leqslant\left|x+\frac{\beta}{\alpha}\right|+t k(1-\Phi(\alpha x+\beta))+\frac{k}{2} \leqslant\left|x+\frac{\beta}{\alpha}\right|+t k+\frac{k}{2} .
\end{aligned}
$$

Пользуясь оценкой $c<\frac{2}{3} \alpha^{2} k$, получаем:

$$
\begin{aligned}
\sup _{0 \leqslant t \leqslant 1} \psi\left(x+t\left(f_{i}(x)-x\right)\right) \leqslant \exp \left\{c\left(\left|x+\frac{\beta}{\alpha}\right|+\frac{3 k}{2}\right)\right\} & \leqslant \\
& \leqslant \exp \left\{\frac{2}{3} \alpha^{2} k\left|x+\frac{\beta}{\alpha}\right|+\alpha^{2} k^{2}\right\} .
\end{aligned}
$$

Следовательно,

$$
\begin{array}{r}
\mathbf{E}\left\{\left|\widetilde{R}_{l}(x)-\widetilde{R}_{l+1}(x)\right|\right\} \leqslant r^{l} \mathbf{E}\left\{|x-f(x)| \exp \left\{\frac{2}{3} \alpha^{2} k\left|x+\frac{\beta}{\alpha}\right|+\alpha^{2} k^{2}\right\}\right\}= \\
=r^{l} \exp \left\{\frac{2}{3} \alpha^{2} k\left|x+\frac{\beta}{\alpha}\right|+\alpha^{2} k^{2}\right\}((1-q)|k \Phi(\alpha x+\beta)|+q|k-k \Phi(\alpha x+\beta)|) \leqslant \\
\leqslant r^{l} k \exp \left\{\frac{2}{3} \alpha^{2} k\left|x+\frac{\beta}{\alpha}\right|+\alpha^{2} k^{2}\right\} .
\end{array}
$$

Просуммировав это выражение по всем $l$ от $n$ до бесконечности:

$$
\mathbf{E} \sum_{l=n}^{+\infty}\left\{\left|\widetilde{R}_{l}(x)-\widetilde{R}_{l+1}(x)\right|\right\} \leqslant \frac{r^{n}}{1-r} k \exp \left\{\frac{2}{3} \alpha^{2} k\left|x+\frac{\beta}{\alpha}\right|+\alpha^{2} k^{2}\right\},
$$

получим оценку

$$
\begin{aligned}
\mathbf{E}\left\{\left|\widetilde{R}_{n}(x)-\widetilde{R}_{\infty}(x)\right|\right\} \leqslant \mathbf{E} \sum_{l=n}^{+\infty}\left\{\left|\widetilde{R}_{l}(x)-\widetilde{R}_{l+1}(x)\right|\right\} \leqslant & \\
& \leqslant \frac{r^{n}}{1-r} k \exp \left\{\frac{2}{3} \alpha^{2} k\left|x+\frac{\beta}{\alpha}\right|+\alpha^{2} k^{2}\right\} .
\end{aligned}
$$

Выражение в правой части конечно для любого $x$, поэтому $\widetilde{R}_{\infty}(x)$ существует и конечен почти наверное.

Повторим теперь те же действия с $\mathbf{E}\left\{\left|\widetilde{R}_{n}(x)-\widetilde{R}_{n}(y)\right|\right\}$ :

$$
\begin{aligned}
& \mathbf{E}\left\{\left|\widetilde{R}_{n}(x)-\widetilde{R}_{n}(y)\right|\right\} \leqslant r^{n} \mathbf{E}\left\{|y-x| \sup _{0 \leqslant t \leqslant 1} \psi(x+t(y-x))\right\}= \\
&=r^{n}|y-x| \sup _{0 \leqslant t \leqslant 1} \psi(x+t(y-x)) .
\end{aligned}
$$

Снова оценим супремум, пользуясь тем, что $c<\frac{2}{3} \alpha^{2} k$. При $0 \leqslant t \leqslant 1$ верно неравенство

$$
\lambda(x+t(y-x))=|| x+t(y-x)+\frac{\beta}{\alpha}\left|-\frac{k}{2}\right| \leqslant\left|x+\frac{\beta}{\alpha}\right|+t|y-x|+\frac{k}{2},
$$


откуда

$$
\begin{aligned}
\sup _{0 \leqslant t \leqslant 1} \psi(x+t(y-x)) \leqslant \exp \left\{c\left(\left|x+\frac{\beta}{\alpha}\right|+|y-x|+\frac{k}{2}\right)\right\} & \leqslant \\
& \leqslant \exp \left\{\frac{2}{3} \alpha^{2} k\left(\left|x+\frac{\beta}{\alpha}\right|+|y-x|\right)+\frac{1}{3} \alpha^{2} k^{2}\right\} .
\end{aligned}
$$

Следовательно,

$$
\mathbf{E}\left\{\left|\widetilde{R}_{n}(x)-\widetilde{R}_{n}(y)\right|\right\} \leqslant r^{n}|y-x| \exp \left\{\frac{2}{3} \alpha^{2} k\left(\left|x+\frac{\beta}{\alpha}\right|+|y-x|\right)+\frac{1}{3} \alpha^{2} k^{2}\right\} .
$$

Устремив $n$ к бесконечности, получаем, что $\widetilde{R}_{\infty}(x)$ не зависит от $x$, что окончательно доказывает наличие единственного стационарного распределения процесса $\left\{R_{n}(x)\right\}$.

Кроме того, объединяя оба полученных неравенства, получаем следующую оценку скорости сходимости $\widetilde{R}_{n}(x)$ :

$$
\begin{aligned}
\mathbf{E}\left\{\left|\widetilde{R}_{n}(x)-\widetilde{R}_{\infty}(y)\right|\right\} \leqslant r^{n} & \exp \left\{\frac{2}{3} \alpha^{2} k\left|x+\frac{\beta}{\alpha}\right|+\frac{1}{3} \alpha^{2} k^{2}\right\} \times \\
& \times\left(\frac{k}{1-r} \exp \left\{\frac{2}{3} \alpha^{2} k^{2}\right\}+|x-y| \exp \left\{\frac{2}{3} \alpha^{2} k|x-y|\right\}\right) .
\end{aligned}
$$

\section{Список литературы}

1. Авдеев В.А., "Стационарное распределение рейтинга игрока в модели Эло с одним соперником", Дискрет. матем., 26:4 (2014), 3-14.

2. Carlsson N., "A contractivity condition for iterated function systems", J. Theor. Probab., 15:3 (2002), 613-630.

3. Феллер В., Введение в теорию вероятностей и ее приложения, т. 1, Мир, 1964.

4. Letac G., "A contraction principle for certain Markov chains and its applications", Random matrices and their applications, Contemp. Math., 50, Amer. Math. Soc., 1986, 263-273.

5. Steinsaltz D., "Locally contractive iterated function systems", Ann. Prob., 27:4 (1999), $1952-1979$. 